\title{
Adverse events after botulinum A toxin injection for neurogenic voiding disorders
}

\author{
K De Laet ${ }^{1}$ and $\mathbf{J}-\mathbf{J}$ Wyndaele*,1 \\ ${ }^{1}$ Department of Urology, Faculty of Medicine, University of Antwerp, Belgium
}

Objectives: To review the side effects of local injections of botulinum A toxin (BTX-A).

Methods: A medline search for publications about adverse events after injection of BTX-A for lower urinary tract dysfunctions.

Results: We found four publications that report generalised side effects after BTX-A injection for detrusor overactivity (two) and detrusor-sphincter dyssynergia (two). The causes of generalised adverse events are not clear, but spread outside the target organ and into the systemic circulation may contribute. The dose used, the injection volume and the injection technique can all play a role.

Conclusion: Generalised side effects after BTX-A injection for voiding disorders are rare but they can be very disabling for spinal cord-injured patients. Although no long-term side effects are reported so far, urologists should be aware that these effects of BTX-A injections are unknown.

Spinal Cord (2005) 43, 397-399. doi:10.1038/sj.sc.3101736; Published online 1 March 2005

Keywords: botulinum toxin; lower urinary tract; voiding disorders; adverse events

\section{Introduction}

Botulinum A toxin (BTX-A) is produced by the bacterium Clostridium botulinum. It is one of the most toxic substances known to humans. ${ }^{1}$ The poisonous properties of BTX-A are due to its inhibition of acetylcholine release at the neuromuscular junction, causing flaccid paralysis. This paralytic effect of BTX-A has been used not only for treating various pathologies like strabismus, cervical dystonia, hyperhidrosis and migraine headaches but also for cosmetic reasons. ${ }^{2}$ Since the first description of BTX-A in urology by Dykstra et $a l^{3}$ many reports have been published about the applications of this neurotoxin in lower urinary tract (LUT) dysfunction. Local injections of BTX-A seem to be successful in treating detrusor-sphincter dyssynergia, neurogenic and idiopathic detrusor overactivity, chronic urinary retention and chronic prostatic pain. ${ }^{4}$ The advantage of an intramuscular injection - compared to an oral treatment with alpha blockers, muscle relaxants or anticholinergics - would be the local effect without systemic action. However, there have been some publications in which generalised side effects after BTX-A injection in the LUT are reported..$^{5-8}$

*Correspondence: Dr J-J Wyndaele, UZA 10 Wilrijkstraat, B 2650 Edegem, Belgium

\section{Methods}

We have searched in the Pubmed online database to find reports about adverse events after BTX-A injections for LUT pathology. The following keywords were used: botulinum toxin, bladder, external urethral sphincter, lower urinary tract, adverse events, complications and general muscle weakness. To complete our search, we checked the references listed in these publications.

\section{Results}

We found four publications reporting adverse events after local injection of BTX-A in the LUT.

Dykstra and Sidi injected 140 U.I. of BTX-A at the first and 240 U.I. at all next sessions in the external urethral sphincter of five spinal cord-injured patients with detrusor-sphincter dyssynergia. Three of them experienced generalised upper extremity weakness after the initial injections. This could be documented on electromyographic studies of the deltoid muscle in one patient. $^{5}$

Del Popolo reported hypostenia with reduced supralesional muscle force in five out of 61 patients with detrusor hyperreflexia after injection of 300 U.I. of Botox $^{\circledR}$ or 1000 U.I. of Dysport ${ }^{\circledR}$ at $20-30$ sites in the detrusor muscle. Moreover, four patients had vision disturbances that disappeared immediately after 
anticholinergic suspension. None of 10 other patients experienced any of these adverse events after receiving 500 U.I. of Dysport ${ }^{\circledR}$. 6

$\mathrm{Kuo}^{7}$ injected $50-100$ U.I. of BTX-A in the external urethral sphincter of 20 patients. One patient had high fever lasting 2 weeks after the injection.

In our own experience, we had two cases of generalised muscle weakness after BTX-A injection for neurogenic detrusor overactivity. One patient received 500 U.I. of Dysport ${ }^{\circledR}$ without effect, but 3 months later she developed general muscle weakness but no increase in bladder capacity after injection of 1000 U.I. The second patient experienced the same complication after injection of 300 U.I. of Botox ${ }^{\circledR}$. In this case, bladder spasticity abolished almost completely. ${ }^{8}$

All side effects were self-limiting and lasted 2 weeks-2 months after the injection.

\section{Discussion}

The following questions come up. Why do side effects occur? And secondly, why only in a number of the patients treated? Technique-related factors may play a role. Usually, the product is injected in the detrusor muscle at different sites, sparing the trigone. Since the thickness of the detrusor also depends on the grade of bladder filling, it would seem not difficult to puncture through the muscle especially at high bladder filling grade. Moreover, between trabeculation bars, the bladder wall can be very thin and so easily perforated. The product would then spread around the bladder and cause an action outside the target organ. Similar effects after BTX-A injections for nonurologic disorders are well known. In a study of 107 patients with torticollis, dysphagia was the most frequent adverse event after intramuscular local injection and could be explained by the local diffusion of BTX-A in the adjacent structures. ${ }^{9}$ To standardise the injection technique and especially to be cautious of the depth of introduction of the needle in the bladder wall does seem important to prevent injection in tissue other than detrusor muscle. Kuo injected the detrusor muscle at a bladder volume of $150 \mathrm{ml}$ and an injection depth of 3-5 mm. According to this study, multiple injections of lower doses of BTX-A appear to play a role in achieving a satisfactory result and in the prevention of systemic side effects. ${ }^{10}$ Suburothelial injection could be a good alternative. Injection of 200 U.I. of BTX-A under the urothelium has been shown to be effective in treating detrusor overactivity of varying aetiology. The effectiveness of BTX-A in the suburothelium is even thought to be better than injection in the detrusor for treating overactive bladder. ${ }^{11}$

The dose injected or the total injected volume could be too high. Borodic et $a l^{12}$ showed that local diffusion of BTX-A in adjacent structures is dose dependent. ${ }^{12} \mathrm{~A}$ hemidystonia patient developed general weakness after a local injection of in total 900 U.I. of Dysport ${ }^{\circledR}$ in arm and hand muscles. Later, six sets of injections with a dose below 600 U.I. gave no more adverse events. ${ }^{13}$ Del
Popolo $^{6}$ reduced the injected dose to half of the initial and saw no more side effects after injection of the detrusor muscle. Kuo spread a total of 200 U.I. of Botox ${ }^{\circledR}$ over 40 sites of the detrusor, while most other researchers used 300 U.I. at 20-30 sites. Perhaps multiple injections of lower doses could play a role in the prevention of side effects, but this needs to be studied. ${ }^{10}$

Another important factor can be the interval between injections. BTX-A binds to specific receptors at the nerve terminal and is then internalised. ${ }^{14}$ Since this binding is irreversible, receptors get saturated and further uptake of BTX-A is impaired. ${ }^{15}$ One of the local reactions will be the development of new nerve sprouts. However, the receptor-binding capacity of BTX-A in these is not clear. At repeated injections, the receptor-binding capacity may be exceeded, which can lead to systemic spread. To prevent accumulation of BTX-A at failure of treatment, a certain interval between subsequent injections is to be respected. Dykstra and Sidi ${ }^{5}$ changed the interval between repeated injections from 1-2 weeks as they were convinced that the transient weakness was caused by a too large cumulative dose. In case of successful treatment, accumulations will unlikely to occur, as the mean effect of local BTX-A injection lasts 2-9 months after treatment of detrusor-sphincter dyssynergia and 9 months after treatment of detrusor overactivity, respectively. ${ }^{4}$

Spread in the nervous structures through retrograde axonal transport might be another possible mechanism of distant muscle weakness. Radioactive-labelled BTXA migrated towards the spinal cord of cats after injection into the gastrocnemius muscle. ${ }^{16}$ In this study, it was not clear whether the radioactivity found in the spinal cord was due to the spread of BTX-A or of its inactive metabolites.

Generalised muscle weakness occurs, not only in urological treatment but also after injection of BTX-A in the neck, arm and foot muscles. ${ }^{13,17}$ This general muscle weakness is usually self-limiting and not life threatening. However, for some patients, it can be temporarily very disabling. Paraplegic patients can become completely dependent when they get upper extremitiy weakness. Tetraplegic patients with high spinal cord lesions may develop difficulties with breathing. Disabled sportsmen can loose their physical condition. ${ }^{8}$

Long-term effects of BTX-A injections remain so far unknown. Even though there were no toxin-related clinical complications in most of the studies published so far after 1-2 years, one should be aware of hidden consequences of repeated injections. That subclinical effects of BTX-A on neuromuscular transmission can appear distant from the injection site has been shown. Olney et $a l^{18}$ found changes in single-fibre EMG on the biceps brachii after neck muscle injection for torticollis. Lange et $a l^{19}$ had the same findings in a double-blind study. Possibly, small amounts of BTX-A entering the systemic circulation can be an explanation for the generalised side effects after local injections. The longterm and cumulative effect has not yet been studied. 
BTX-A is known to cause chemodenervation. Reinnervation occurs as new nerve sprouts grow. The longterm effect of this on the function of the bladder remains uncertain. Harper et al gave hypothetical concerns that repeated injections may eventually enhance the pathological innervation leading to tolerance and even exacerbation of local symptoms. Repeated injections could cause muscle fibrosis after several years though such effect has not been shown in shorter follow-up so far. ${ }^{20}$

Response to BTX-A injections over the years could get lower by the development of antibodies though this has been thought to be mainly related to the proteins in elderly preparations. ${ }^{21}$ It would create a very difficult situation if at one stage the bladder would not respond any more to BTX-A treatment because of antibody formation, while the bladder had become even more unstable because of pathologic reïnnervation.

The clinician should also be aware that effects of BTXA might be more permanent than expected. Kranjc et $a l^{22}$ saw long-term morphologic and functional changes after BTX-A injection of ocular rectus muscles in rats with no recovery of muscle contraction. The effect of BTX-A on striated and smooth muscle of the LUT should be studied.

\section{Conclusions}

Although local injections with BTX-A seem to have excellent short-term results in treating patients with different kinds of LUT dysfunction, urologists should be aware of systemic side effects. Although these adverse events are self-limiting and not life threatening, they can be very disabling for some patients, especially for spinal cord-injured patients. In order to avoid these complications, optimal dose, injection volume and injection techniques should be studied further. Since the longterm effects of repeated injections are not known, some caution in the use of BTX-A is advocated.

\section{References}

1 Lamanns C. The most poisonous poison. Science 1969; 130: $763-772$.

2 Klein AW. Contraindications and complications with the use of botulinum toxin. Clin Dermatol 2004; 22: 66-75.

3 Dykstra DD, Sidi AA, Scott AB, Pagel JM, Goldish GD. Effects of botulinum A toxin on detrusor-sphincter dyssynergia in spinal cord injury patients. J Urol 1988; 139: $919-922$.

4 Leippold T, Reitz A, Schurch B. Botulinum toxin as a new therapy option for voiding disorders: current state of the art. Eur Urol 2003; 44: 165-174.

5 Dykstra DD, Sidi AA. Treatment of detrusor-sphincter dyssynergia with botulinum A toxin: a double-blind study. Arch Phys Med Rehabil 1990; 71: 24-26.
6 Del Popolo G. Botulinum-A toxin in the treatment of detrusor hyperreflexia. Neurourol Urodyn 2001; 20: $522-524$.

7 Kuo HC. Effectiveness of periurethral botulinum toxin injection in the treatment of voiding dysfunction due to detrusor underactivity and non-relaxing urethra. Neurourol Urodyn 2002; 21: 387-388.

8 Wyndaele JJ, Van Dromme SA. Muscular weakness as side effect of botulinum toxin injection for neurogenic detrusor overactivity. Spinal Cord 2002; 40: 599-600.

9 Anderson TJ et al. Botulinum toxin treatment of spasmodic torticollis. J R Soc Med 1992; 85: 524-529.

10 Kuo HC. Urodynamic evidence of effectiveness of botulinum A toxin injection in treatment of detrusor overactivity refractory to anticholinergic agents. Urology 2004; 63: 868-872.

11 Liu H, Kuo HC. Clinical Effectiveness of Suburothelial Injection of Botulinum A Toxin in the Treatment of Refractory Detrusor Overactivity. International Continence Society annual meeting, Paris 2004. Abstract 380.

12 Borodic GE, Ferrante R, Pearce LB, Smith K. Histologic assessement of dose-related diffusion and muscle fiber response after therapeutic botulinum A toxin injections. Mov Disord 1994; 9: 31-39.

13 Bhatia KP et al. Generalised muscular weakness after botulinum toxin injections for dystonia: a report of three cases. J Neurol Neurosurg Psychiarty 1999; 67: 90-93.

14 Dolly JO, Black J, Williams RS, Melling J. Acceptors for botulinum neurotoxin reside on motor nerve terminals and mediate its internalisation. Nature 1984; 307: 457-460.

15 Hambleton P, Moore AP. Botulinum neurotoxin: origin, structure, molecular actions and antibodies. In: Moore P (ed). Handbook of Botulinum Toxin Treatment. Blackwell Science: Oxford 1995, pp 16-27.

16 Wiegand H, Erdmann G, Wellhoner HH. 125I-labelled botulinum A neurotoxin: pharmacokinetics in cats after intramuscular injection. Naunyn Schmiedebergs Arch Pharmacol 1976; 292: 161-165.

17 Bakheit AM, Ward CD, McLellan DL. Generalised botulism-like syndrome after intramuscular injections of botulinum toxin type A: a report of two cases. J Neurol Neurosurg Psychiatry 1997; 62: 198.

18 Olney RK, Aminoff MJ, Gelb DJ, Lowenstein DH. Neuromuscular effects distant from the site of botulinum neurotoxin injection. Neurology 1988; 38: 1780-1783.

19 Lange DJ et al. Distant effects of locally injected botulinum toxin: a double-blind study of single fiber EMG changes. Muscle Nerve 1991; 14: 672-675.

20 Harper M, Fowler CJ, Dasgupta P. Botulinum toxin and its applications in the lower urinary tract. Br J Urol 2004; 93: 702-706.

21 Jankovic J, Schwartz K. Response and immunoresistance to botulinum toxin injections. Neurology 1995; 45: 1743-1746.

22 Kranjc BS, Sketelj J, D'Albis A, Erzen I. Long term changes in myosin heavy chain composition after botulinum toxin A injection into rat medial rectus muscle. Invest Ophtalmol Vis Sci 2001; 42: 1358-1364. 